\title{
Effect of passive exposure to cigarette smoke on blood pressure in children and adolescents: a meta-analysis of epidemiologic studies
}

\author{
Mahshid Aryanpur', Mahmoud Yousefifard², Alireza Oraii ${ }^{3}$, Gholamreza Heydari', Mehdi Kazempour-Dizaji ${ }^{4}$, \\ Hooman Sharifi ${ }^{1}$, Mostafa Hosseini ${ }^{5^{*}}$ and Hamidreza Jamaati ${ }^{1}$
}

\begin{abstract}
Background: Hypertension is an emerging disease in children and adolescents resulting in future morbidities. Cigarette smoking is one of the most studied contributing factors in this regard; however, there are contradictory results among different studies. Therefore, the present meta-analysis tends to assess the relationship between passive exposure to cigarette smoke and blood pressure in children and adolescents.

Method: Medline, Embase, Scopus, EBSCO, and Web of Sciences were systematically reviewed for observational studies up to May, 2017, in which the relationship between cigarette smoking and hypertension were assessed in children and adolescents. The meta-analysis was performed with a fixed effect or random effects model according to the heterogeneity.

Results: Twenty-nine studies were included in present meta-analysis incorporating 192,067 children and adolescents. Active smoking (pooled $\mathrm{OR}=0.92 ; 95 \% \mathrm{Cl}: 0.79$ to 1.05 ) or passive exposure to cigarette smoke (pooled $\mathrm{OR}=1.01 ; 95 \% \mathrm{Cl}: 0.93$ to 1.10 ) were not associated with developing hypertension in the study population. Despite the fact that active cigarette smoking did not significantly affect absolute level of systolic and diastolic blood pressure, it was shown that passive exposure to cigarette smoke leads to a significant increase in absolute level of systolic blood pressure (pooled coefficient $=0.26 ; 95 \% \mathrm{Cl}: 0.12$ to 0.39 ).
\end{abstract}

Conclusion: Both active and passive cigarette smoking were not associated with developing hypertension in children and adolescents. However, passive cigarette smoke was associated with higher level of systolic blood pressure in children and adolescents.

Keywords: Hypertension, Blood pressure, Children and adolescent, Smoking

\section{Background}

Hypertension has been named "Silent Killer" by some researchers as it is a disease that can lead to cardiovascular disorders, cerebral infarction and renal failure [1]. About $1-3 \%$ of children have hypertension [2] which has a secondary etiology in about $80 \%$ of cases and is a consequence of an underlying factor such as family history, body mass index, socioeconomic status and nutritional

\footnotetext{
* Correspondence: mhoosein110@yahoo.com

${ }^{5}$ Department of Epidemiology and Biostatistics, School of Public Health,

Tehran University of Medical Sciences, Poursina Ave, Tehran, Iran

Full list of author information is available at the end of the article
}

status [3-5]. Some studies have reported that cigarette smoking is a risk factor for hypertension. There are strong evidence that exposure to cigarette smoke has adverse effects on health during childhood, adolescence and even adulthood [6-8]. Studies show that children exposed to cigarette smoke during fetal life have significantly lower birth weights in addition to higher risk of getting overweight or obese in future [9]. Moreover, active smoking or passive exposure to cigarette smoke cause dysfunction of capillary endothelium in healthy individuals suggesting an association between cigarette smoking and hypertension [10]. However, some studies

(c) The Author(s). 2019 Open Access This article is distributed under the terms of the Creative Commons Attribution 4.0 International License (http://creativecommons.org/licenses/by/4.0/), which permits unrestricted use, distribution, and reproduction in any medium, provided you give appropriate credit to the original author(s) and the source, provide a link to the Creative Commons license, and indicate if changes were made. The Creative Commons Public Domain Dedication waiver (http://creativecommons.org/publicdomain/zero/1.0/) applies to the data made available in this article, unless otherwise stated. 
report that there is no association between cigarette smoking and hypertension in children [11].

The importance of this issue is that both cigarette smoking and hypertension are two risk factors of non-communicable diseases [12, 13]. Therefore, presence of two risk factors in a single individual may lead to an additive or synergistic effect on incidence of chronic diseases. This issue must be more emphasized in childhood as most diseases of adulthood are consequences of childhood health status.

Multiple studies have been conducted regarding the association between exposure to cigarette smoke and hypertension in recent years in the field of pediatrics. However, contradictory results were reported in various studies. Hence, the present meta-analysis was designed to assess the association between exposure to cigarette smoke and systolic and diastolic blood pressure in addition to its risk for incidence of hypertension in children and adolescents.

\section{Methods}

\section{Study design}

The present study is designed based on instructions of Meta-analysis of Observational Studies in Epidemiology (MOOSE) statement [14]. All cohort, case-control and cross sectional studies on children and adolescents between the ages of 0 and 18 years old assessing the relation of exposure to cigarette smoke and hypertension were reviewed. Exclusion criteria were combination of results with data of adults, lack of adjustment for potential confounders, review articles and lack of reported odds ratio (OR) or regression coefficient (Beta).

\section{Search strategy}

In the present study, an extensive search was performed in electronic databases of Medline (via PubMed),
Embase, Scopus, EBSCO, and Web of Sciences until the end of May, 2017. Keywords were selected using databases of Mesh and Emtree and with the help of specialists in fields of hypertension and cigarette smoking. These keywords were phrases related to usage or exposure to cigarette smoke and hypertension. Search query in Medline is shown in Table 1. In addition, a manual search was done in the bibliography of related articles, contact was made with authors of related articles and at the end a search in the thesis division of the ProQuest database to screen additional articles and unpublished data. Additionally, Google search engine and Google scholar were also used to find Grey literature.

\section{Data extraction and quality assessment}

Data extraction method is reported in our previous meta-analyses [15-24]. Search records were pooled and the duplicated studies were removed using EndNote software (version X5, Thomson Reuters, 2011). Two independent researchers screened titles and abstracts and potentially relevant studies were reviewed more precisely. Any disagreement was resolved by discussion with a third reviewer. Relevant studies were summarized including their data regarding study design, population characteristics (age and sex), sample size, outcome (hypertension, levels of systolic and diastolic blood pressure), blinding status, data gathering method (consecutive, convenience), study design (cohort, cross sectional or case-control) and possible bias. The data gathering form was designed based on instructions of PRISMA statement [25].

In the present study, two separate experiments were entered in the study if data were differentiated by sex. When regression models with different adjustments were reported, the analysis with highest number of adjustments was entered. In addition, if results were shown

Table 1 Search strategy of present study in Medline

\begin{tabular}{|c|c|}
\hline Databases & Search query \\
\hline $\begin{array}{l}\text { Medline (via } \\
\text { PubMed) }\end{array}$ & 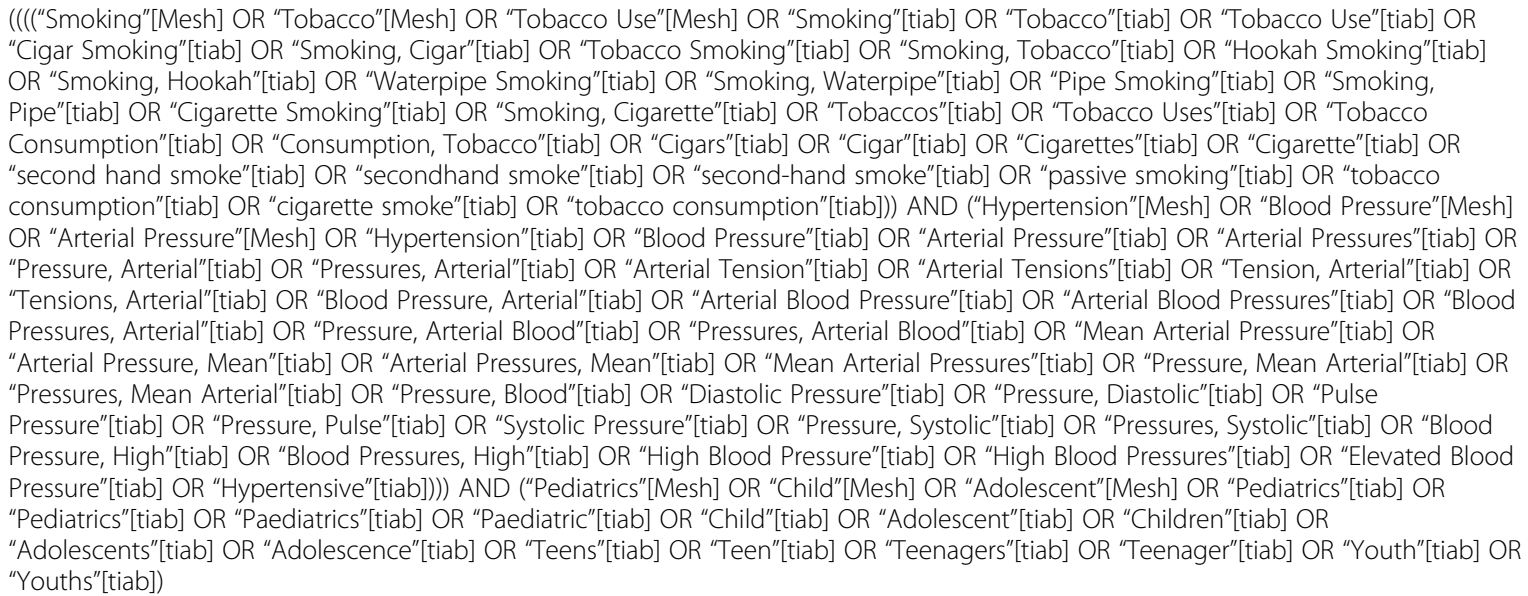 \\
\hline
\end{tabular}


in graphs, the methods proposed by Sistrom and Mergo for data extraction from graphs were used [26].

At the end, study quality assessment was done using suggested instructions of Newcastle-Ottawa Scale [27]. Hence, quality of different studies was assessed based on following criteria: 1) Is the case definition adequate, 2) Representativeness of the cases, 3) Definition of controls, 4) Comparability, 5) Ascertainment of exposure, 6) Same method ascertainment case control and 7) Reporting Non-Response rate.

\section{Statistical analyses}

Data were analysed by STATA 14.0. Analyses were done in two steps. In first step, the association between active smoking and passive exposure to cigarette smoke with hypertension in childhood and adolescence were assessed. Only studies were entered in this step which had defined hypertension as systolic or diastolic blood pressure more than 95 percentile. Hence, data were entered as adjusted OR and 95\% confidence interval (95\% CI).

In second step, the association between active smoking and passive exposure to cigarette smoke with absolute value of systolic and diastolic blood pressure were assessed. The related data for mentioned analysis were entered as adjusted regression coefficient (Beta) and 95\% CI. The association between active smoking and hypertension was reported separately from passive exposure in all analyses. Additionally, the association between active and passive smoking with blood pressure was reported for systolic and diastolic blood pressure, separately.

Data were pooled in all analyses and an overall effect size and 95\% CI were reported. Heterogeneity among studies was assessed using $\mathrm{I}^{2}$ test $\left(\mathrm{I}^{2}\right.$ greater than $50 \%$ or $p$ value less than 0.1 were defined as heterogeneous). Fixed effect method was used in homogenous studies and random effect model was used in case of heterogeneous studies. Subgroup analyses were done to find the source of heterogeneity which included type of study (cohort, cross sectional), age group of children under study, definition of smoker, exposure period (before birth or domestic use), parental smoking habit (mother, father and both) and sample size (less than 1000 patients and equal or greater than 1000). In addition, Egger's test was used to assess publication bias. A $p$ value of less than 0.05 was defined significant in all analyses.

\section{Results}

\section{Characteristics of entered studies}

Eight thousand three hundred ninety-two records were gathered in the primary search. After omitting the duplicated articles and primary screening, 92 potentially relevant studies were found. At the end, 29 articles were entered in the present study after assessing their full texts [28-57] (Fig. 1). Data of 192,067 children and adolescence between the ages of 3 and 18 years old were assessed. Boys comprised $75.77 \%$ of patients. 12 cohorts, 16 cross-sectional and 1 case-control studies were entered.

Fifteen studies evaluated the association between active smoking or passive exposure to cigarette smoke with hypertension [28-42] and 17 studies assessed the association between cigarette smoking and absolute levels of systolic and diastolic blood pressure [34, 41-56]. Three of the mentioned studies assessed both types of outcome [34, 41, 42]. One of these studies was in Portuguese [35] and another one was in Korean [47].

Fifteen studies assessed the association between active smokers [28-31, 33, 35-39, 47, 48, 50, 51, 56] and 16 studies assessed the association between passive exposure to cigarette smoke [32, 34, 37, 40-46, 49, 52-56] and hypertension or absolute levels of blood pressure. Two studies assessed both types of exposure [37, 56]. 13 studies assessed the exposure during pregnancy [37, 4044, 46, 49, 52-56], 2 studies assessed domestic exposure (after pregnancy) [32, 34] and 3 studies assessed both mentioned passive exposures [41, 42, 56].

There were different definitions of smoking among studies and in 7 studies there was no standard definition for smoker. In 7 studies being a smoker was only asked and answered with a yes/no question [35, 38, 40, 41, 45, 50, 54]. In 11 studies, the individuals were asked if they were current smoker or if they have smoked during pregnancy [31-33, 39, 42-44, 46, 47, 52, 53]. Table 2 and Table 3 show characteristics of entered studies.

\section{Quality assessment of studies}

Quality assessment of studies is depicted in Fig. 2. As shown, ascertainment of exposure is biased in most studies. Other items were in appropriate levels in most studies.

\section{Meta-analysis \\ Effect of cigarette smoking on hypertension}

\section{Active smoking}

In the present meta-analysis, 10 studies assessed the association between active smoking and hypertension. Results were reported for boys and girls separately in the study of Dasgupta et al. [33]. Hence, the mentioned study is entered as two separate experiments. Analyses confirmed homogeneity of studies $\left(\mathrm{I}^{2}=0.0 \% ; p=0.53\right)$. Additionally, publication bias was not observed in analyses (Coefficient $=1.50$; $p=0.69$ ).

Pooled analysis showed that active smoking in childhood was not associated with developing hypertension in children and adolescents (pooled OR $=0.92 ; 95 \% \mathrm{CI}$ : 0.79 to 


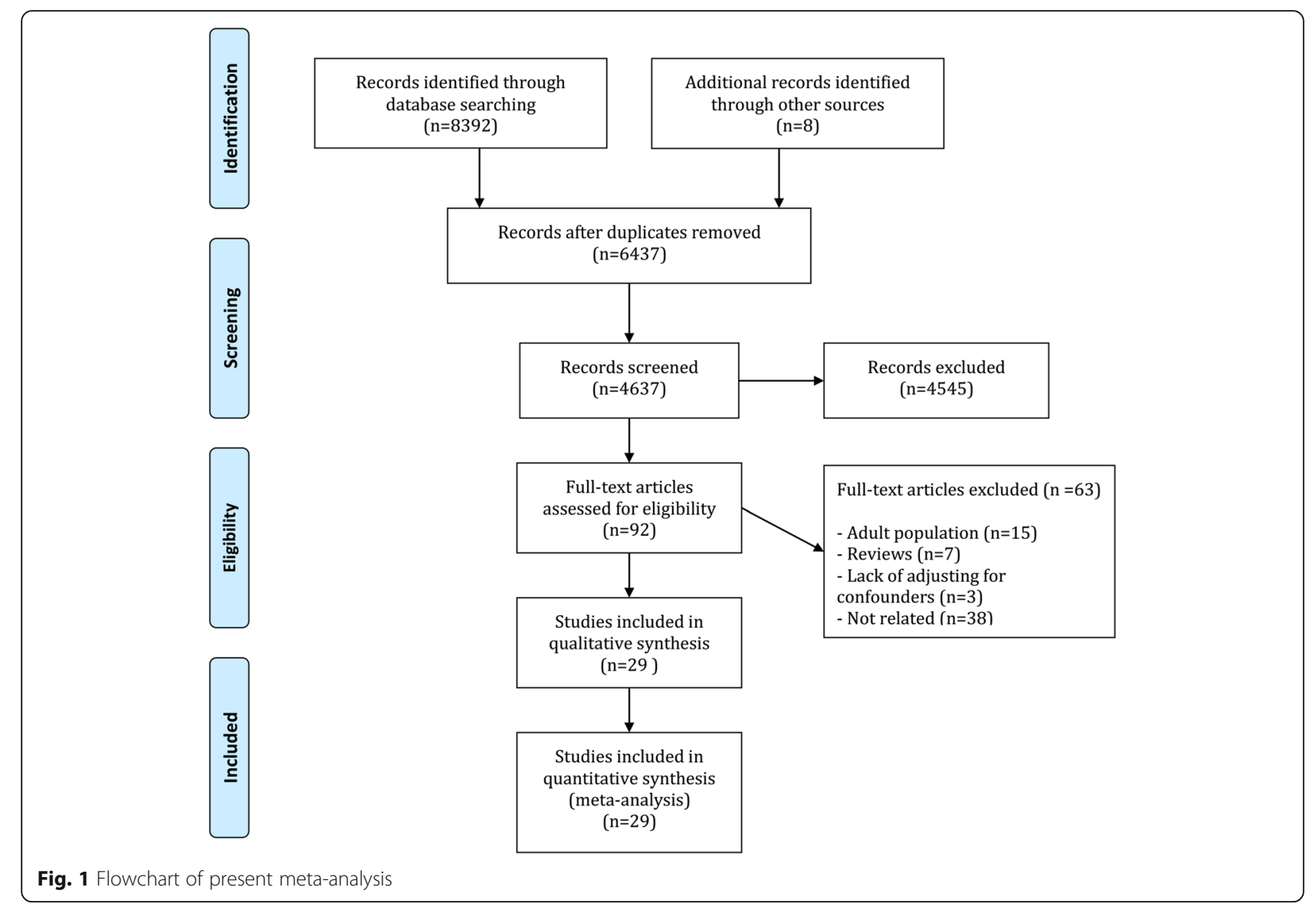

1.05). Subgroup analysis was not needed as heterogeneity was not found at this section (Fig. 3a).

\section{Passive exposure to cigarette smoke}

7 studies were entered in order to assess the association between passive exposure to cigarette smoke and hypertension. One study assessed passive exposure in pregnancy and domestic use [37]. Hence, the mentioned study was entered in the study as two separate experiments. Heterogeneity $\left(\mathrm{I}^{2}=36.7 \% ; p=0.12\right)$ and publication bias (Coefficient $=1.66 ; p=0.80$ ) were not present in analyses. Pooled analyses showed that passive exposure to cigarette smoke was not associated with developing hypertension in children and adolescents (pooled OR $=1.01$; 95\% CI: 0.93 to 1.10 ) (Fig. 3a).

There were two types of passive exposure to cigarette smoke among studies including exposure during pregnancy and domestic use after pregnancy. Therefore, effects of mentioned exposures were assessed separately.

Exposure to cigarette smoke during fetal period and its association with developing hypertension

In children with passive exposure during pregnancy, exposure to cigarette smoke in fetal period did not have a significant effect on hypertension in childhood and adolescence $(\mathrm{OR}=0.99$; 95\% CI: 0.85 to 1.13$)$. Results of this section are depicted in Fig. 3b. As shown, heterogeneity $\left(\mathrm{I}^{2}=\right.$ 24.4\%; $p=0.26$ ) and publication bias (Coefficient $=3.50 ; p=$ 0.61 ) were not observed.

\section{Effect of domestic exposure to cigarette smoke on hypertension}

It was shown that domestic exposure (after fetal period) to cigarette smoke was not associated with developing hypertension $(\mathrm{OR}=1.05$; $95 \% \mathrm{CI}: 0.81$ to 1.29$)$. Additionally, heterogeneity $\left(\mathrm{I}^{2}=45.7 \% ; p=0.14\right)$ and publication bias (coefficient $=-15.8 ; p=0.29$ ) was not observed in this section (Fig. 3b).

\section{Effect of cigarette smoking on absolute level of systolic and diastolic blood pressure}

\section{Effect of active cigarette smoking on level of systolic blood pressure}

Results of this section are depicted in Fig. 4. Analyses in this section were done based on random effect model due to heterogeneity among studies ( $\mathrm{I} 2=53.3 \% ; p=0.07)$. At the end, it was shown that active cigarette smoking does not 
Table 2 Summery of included studies which reported the relationship of pediatric hypertension (HTN) and smoking

\begin{tabular}{|c|c|c|c|c|c|c|c|c|c|}
\hline $\begin{array}{l}\text { Author, year; } \\
\text { country }\end{array}$ & $\begin{array}{l}\text { Type of } \\
\text { survey }\end{array}$ & $\begin{array}{l}\text { Study } \\
\text { type }\end{array}$ & $\begin{array}{l}\text { Total } \\
\text { sample }\end{array}$ & $\operatorname{Sex}^{a}$ & Age & $\begin{array}{l}\text { HTN } \\
\text { definition }^{b}\end{array}$ & $\begin{array}{l}\text { Smoking } \\
\text { definition }\end{array}$ & $\begin{array}{l}\text { Type of } \\
\text { exposer }\end{array}$ & BP measurements method \\
\hline $\begin{array}{l}\text { Akis, 2009; } \\
\text { Turkey [28] }\end{array}$ & Local & $\begin{array}{l}\text { Case- } \\
\text { control }\end{array}$ & 236 & 42 & $\begin{array}{l}12 \\
\text { to } \\
14\end{array}$ & $\mathrm{BP}>95$ th & $\begin{array}{l}\text { More than } 1 \\
\text { cigarette per } \\
\text { week }\end{array}$ & Active & $\begin{array}{l}\text { Three times measurement of BP } \\
\text { using an automatic } \\
\text { sphygmomanometer device }\end{array}$ \\
\hline $\begin{array}{l}\text { Bozza, 2016; } \\
\text { Brazil [29] }\end{array}$ & Local & $\begin{array}{l}\text { Cross- } \\
\text { section }\end{array}$ & 1242 & 596 & $\begin{array}{l}11 \\
\text { to } \\
17\end{array}$ & $\mathrm{BP}>95$ th & $\begin{array}{l}\text { Cigarettes } \\
\text { smoked } 10 \text { to } 30 \text { days }\end{array}$ & Active & $\begin{array}{l}\text { Two times measurement of BP } \\
\text { using auscultatory method }\end{array}$ \\
\hline $\begin{array}{l}\text { Christofaro, } \\
\text { 2015; Brazil [30] }\end{array}$ & Local & $\begin{array}{l}\text { Cross- } \\
\text { section }\end{array}$ & 1231 & NR & $\begin{array}{l}14 \\
\text { to } \\
17\end{array}$ & $\mathrm{BP}>95$ th & $\begin{array}{l}\text { Current daily } \\
\text { smoking at } \\
\text { least } 1 \\
\text { cigarette }\end{array}$ & Active & $\begin{array}{l}\text { two times measurement of BP } \\
\text { using an automatic oscillometric } \\
\text { device }\end{array}$ \\
\hline $\begin{array}{l}\text { Cinteza, 2013; } \\
\text { Romania [31] }\end{array}$ & Regional & $\begin{array}{l}\text { Cross- } \\
\text { section }\end{array}$ & 4886 & 2407 & $\begin{array}{l}3 \text { to } \\
17\end{array}$ & $\mathrm{BP}>95$ th & $\begin{array}{l}\text { Current } \\
\text { smoking }\end{array}$ & Active & $\begin{array}{l}\text { Three times measurement of BP. } \\
\text { First measurement using an } \\
\text { automatic oscillometric device } \\
\text { and a BP mercury device for the } \\
\text { second and the third measurement }\end{array}$ \\
\hline $\begin{array}{l}\text { Crispim, 2014; } \\
\text { Brazil [32] }\end{array}$ & Local & $\begin{array}{l}\text { Cross- } \\
\text { section }\end{array}$ & 276 & 145 & $\begin{array}{l}2 \text { to } \\
4\end{array}$ & $\mathrm{BP}>95$ th & $\begin{array}{l}\text { Current } \\
\text { smoking }\end{array}$ & $\begin{array}{l}\text { Passive } \\
\text { (domestic) }\end{array}$ & $\begin{array}{l}\text { Two times measurement of BP using } \\
\text { a semi-automatic an oscillometric } \\
\text { device }\end{array}$ \\
\hline $\begin{array}{l}\text { Dasgupta, 2006; } \\
\text { Canada [33] }\end{array}$ & Local & Cohort & 1267 & 1018 & $\begin{array}{l}10 \\
\text { to } \\
18\end{array}$ & BP $>90$ th & $\begin{array}{l}\text { Current } \\
\text { smoking }\end{array}$ & Active & $\begin{array}{l}\text { Three times measurement of BP } \\
\text { using an automatic oscillometric } \\
\text { device }\end{array}$ \\
\hline $\begin{array}{l}\text { Giussani, 2013; } \\
\text { Italy [34] }\end{array}$ & Regional & $\begin{array}{l}\text { Cross- } \\
\text { section }\end{array}$ & 1310 & 682 & $\begin{array}{l}5 \text { to } \\
14\end{array}$ & BP $>90$ th & $\begin{array}{l}\text { At least one } \\
\text { parent with } \\
\text { smoking } \\
\text { habit }\end{array}$ & $\begin{array}{l}\text { Passive } \\
\text { (domestic) }\end{array}$ & $\begin{array}{l}\text { Two times measurement of BP } \\
\text { using a aneroid } \\
\text { sphygmomanometer device }\end{array}$ \\
\hline $\begin{array}{l}\text { Gomes, 2009; } \\
\text { Brazil [35] }\end{array}$ & Local & $\begin{array}{l}\text { Cross- } \\
\text { section }\end{array}$ & 1875 & 718 & $\begin{array}{l}14 \\
\text { to } \\
20\end{array}$ & $\mathrm{BP}>95$ th & NR & Active & $\begin{array}{l}\text { Single measurement of BP using an } \\
\text { automatic oscillometric device }\end{array}$ \\
\hline $\begin{array}{l}\text { Guo, 2011; } \\
\text { China [36] }\end{array}$ & Local & $\begin{array}{l}\text { Cross- } \\
\text { section }\end{array}$ & 4445 & 2298 & $\begin{array}{l}5 \text { to } \\
18\end{array}$ & $\mathrm{BP}>95$ th & $\begin{array}{l}\text { At least } 1 \\
\text { cigarette per } \\
\text { month }\end{array}$ & Active & $\begin{array}{l}\text { Two times measurement of BP using } \\
\text { a mercury sphygmomanometer } \\
\text { device }\end{array}$ \\
\hline $\begin{array}{l}\text { International } \\
\text { Collaborative } \\
\text { Group, 1984; } \\
\text { Europe [37] }\end{array}$ & International & Cohort & 2704 & NR & 14 & $\mathrm{BP}>95$ th & $\begin{array}{l}\text { More than } 5 \\
\text { cigarette per } \\
\text { week }\end{array}$ & $\begin{array}{l}\text { Active; } \\
\text { pregnancy }\end{array}$ & $\begin{array}{l}\text { Three times measurement of } \mathrm{BP} \\
\text { using a mercury sphygmomanometer } \\
\text { device }\end{array}$ \\
\hline $\begin{array}{l}\text { Nur, 2008; } \\
\text { Turkey [38] }\end{array}$ & Local & $\begin{array}{l}\text { Cross- } \\
\text { section }\end{array}$ & 1020 & 593 & $\begin{array}{l}14 \\
\text { to } \\
18\end{array}$ & $\mathrm{BP}>95$ th & NR & Active & $\begin{array}{l}\text { Three times measurement of BP } \\
\text { using a mercury sphygmomanometer } \\
\text { device }\end{array}$ \\
\hline $\begin{array}{l}\text { Pileggi, 2005; } \\
\text { Italy [39] }\end{array}$ & Local & $\begin{array}{l}\text { Cross- } \\
\text { section }\end{array}$ & 603 & 284 & $\begin{array}{l}6 \text { to } \\
18\end{array}$ & $\mathrm{BP}>95$ th & $\begin{array}{l}\text { Current } \\
\text { smoking }\end{array}$ & Active & $\begin{array}{l}\text { Three times measurement of } \mathrm{BP} \\
\text { using a mercury sphygmomanometer } \\
\text { device }\end{array}$ \\
\hline $\begin{array}{l}\text { Shankaran, 2006; } \\
\text { USA [40] }\end{array}$ & Regional & Cohort & 516 & 275 & 6 & $\mathrm{BP}>95$ th & NR & Pregnancy & $\begin{array}{l}\text { Two times measurement of BP using } \\
\text { an automatic oscillometric device }\end{array}$ \\
\hline $\begin{array}{l}\text { Simonetti, 2011; } \\
\text { Germany [42] }\end{array}$ & National & $\begin{array}{l}\text { Cross- } \\
\text { section }\end{array}$ & 4236 & 2181 & $\begin{array}{l}4 \text { to } \\
7.5\end{array}$ & $\mathrm{BP}>95$ th & $\begin{array}{l}\text { Current } \\
\text { smoking }\end{array}$ & $\begin{array}{l}\text { Passive } \\
\text { (domestic) }\end{array}$ & $\begin{array}{l}\text { Three times measurement of BP } \\
\text { using an auscultatory aneroid } \\
\text { sphygmomanometry } \\
\text { device }\end{array}$ \\
\hline $\begin{array}{l}\text { van den Berg, } \\
\text { 2013; } \\
\text { Netherland [41] }\end{array}$ & Local & Cohort & 3024 & 1521 & $\begin{array}{l}5 \text { to } \\
6\end{array}$ & $\mathrm{BP}>$ 90th & NR & $\begin{array}{l}\text { Passive } \\
\text { (domestic) }\end{array}$ & $\begin{array}{l}\text { Two or three times measurement of } \\
\text { BP using an automatic } \\
\text { sphygmomanometer device }\end{array}$ \\
\hline
\end{tabular}

\footnotetext{
a Male sex (number of children):
}

${ }^{\mathrm{b}}$ Hypertension (HTN) was defined as systolic or diastolic blood pressure more than 95th percentile; Prehypertension was defined as systolic or diastolic blood pressure between 90th and 95th percentiles

$B P$ Blood pressure, NA Not applicable, NR Not reported 
Table 3 Summery of included studies which reported the relationship of pediatric blood pressure and smoking

\begin{tabular}{|c|c|c|c|c|c|c|c|c|c|}
\hline $\begin{array}{l}\text { Author, year; } \\
\text { country }\end{array}$ & $\begin{array}{l}\text { Type of } \\
\text { survey }\end{array}$ & $\begin{array}{l}\text { Study } \\
\text { type }\end{array}$ & $\begin{array}{l}\text { Total } \\
\text { sample }\end{array}$ & $\operatorname{Sex}^{\mathrm{a}}$ & Age & $\begin{array}{l}\text { Type } \\
\text { of BP }\end{array}$ & $\begin{array}{l}\text { Smoking } \\
\text { definition }\end{array}$ & $\begin{array}{l}\text { Type of } \\
\text { exposer }\end{array}$ & BP measurements method \\
\hline $\begin{array}{l}\text { Belfort, 2012; USA } \\
\text { [43] }\end{array}$ & Local & Cohort & 694 & NR & 6.5 & SBP & $\begin{array}{l}\text { Smoking during } \\
\text { pregnancy }\end{array}$ & Pregnancy & $\begin{array}{l}\text { Three times measurement of BP using an } \\
\text { automatic oscillometric device }\end{array}$ \\
\hline $\begin{array}{l}\text { Blake, 2000; } \\
\text { Australia [44] }\end{array}$ & Regional & Cohort & 702 & NR & 6 & SBP & $\begin{array}{l}\text { Smoking at } 18 \\
\text { weeks gestation }\end{array}$ & Pregnancy & $\begin{array}{l}\text { Two times measurement of BP using a } \\
\text { semi-automatic oscillometric device }\end{array}$ \\
\hline $\begin{array}{l}\text { Brambilla, 2015; } \\
\text { Italy [45] }\end{array}$ & National & $\begin{array}{l}\text { Cross- } \\
\text { section }\end{array}$ & 1294 & NR & $\begin{array}{l}7 \\
\text { to } \\
13\end{array}$ & $\begin{array}{l}\text { SBP } \\
\text { and } \\
\text { DBP }\end{array}$ & NR & $\begin{array}{l}\text { Passive } \\
\text { (domestic) }\end{array}$ & $\begin{array}{l}\text { Three times measurement of BP using a } \\
\text { manual sphygmomanometer device }\end{array}$ \\
\hline $\begin{array}{l}\text { Brion, 2007; UK } \\
\text { [46] }\end{array}$ & Local & Cohort & 6509 & 3281 & 7.7 & $\begin{array}{l}\text { SBP } \\
\text { and } \\
\text { DBP }\end{array}$ & $\begin{array}{l}\text { Smoking at } 18 \\
\text { weeks gestation }\end{array}$ & Pregnancy & $\begin{array}{l}\text { Two times measurement of BP using an } \\
\text { automatic oscillometric device }\end{array}$ \\
\hline $\begin{array}{l}\text { Byeon, 2007; } \\
\text { South Korea [47] }\end{array}$ & Local & $\begin{array}{l}\text { Cross- } \\
\text { section }\end{array}$ & 127 & 82 & $\begin{array}{l}10 \\
\text { to } \\
13\end{array}$ & $\begin{array}{l}\text { SBP } \\
\text { and } \\
\text { DBP }\end{array}$ & Current smoking & Active & $\begin{array}{l}\text { Three times measurement of BP using an } \\
\text { automatic oscillometric device }\end{array}$ \\
\hline $\begin{array}{l}\text { Garoufi, 2017; } \\
\text { Greece [48] }\end{array}$ & Local & $\begin{array}{l}\text { Cross- } \\
\text { section }\end{array}$ & 736 & 366 & $\begin{array}{l}12 \\
\text { to } \\
18\end{array}$ & $\begin{array}{l}\text { SBP } \\
\text { and } \\
\text { DBP }\end{array}$ & $\begin{array}{l}\text { Smoking for at } \\
\text { least } 1 \text { month }\end{array}$ & Active & $\begin{array}{l}\text { Three times measurement of BP using an } \\
\text { automatic oscillometric device }\end{array}$ \\
\hline $\begin{array}{l}\text { Giussani, 2013; } \\
\text { Italy [34] }\end{array}$ & Regional & $\begin{array}{l}\text { Cross- } \\
\text { section }\end{array}$ & 1310 & 682 & $\begin{array}{l}5 \\
\text { to } \\
14\end{array}$ & SBP & $\begin{array}{l}\text { Having one } \\
\text { parent with } \\
\text { smoking habit }\end{array}$ & $\begin{array}{l}\text { Passive } \\
\text { (domestic) }\end{array}$ & $\begin{array}{l}\text { Two times measurement of BP using a } \\
\text { aneroid sphygmomanometer device }\end{array}$ \\
\hline $\begin{array}{l}\text { Hogberg, 2012; } \\
\text { Sweden [49] }\end{array}$ & National & Cohort & 92,730 & 92,730 & $\begin{array}{l}17 \\
\text { to } \\
19\end{array}$ & $\begin{array}{l}\text { SBP } \\
\text { and } \\
\text { DBP }\end{array}$ & $\begin{array}{l}\text { At least } 1 \\
\text { cigarette per day }\end{array}$ & Pregnancy & $\begin{array}{l}\text { Single measurement of BP using automatic } \\
\text { and manual sphygmomanometer devices }\end{array}$ \\
\hline $\begin{array}{l}\text { Katona, 2010; } \\
\text { Hungary [50] }\end{array}$ & Local & $\begin{array}{l}\text { Cross- } \\
\text { section }\end{array}$ & 10,194 & 5163 & 16.6 & $\begin{array}{l}\text { SBP } \\
\text { and } \\
\text { DBP }\end{array}$ & NR & Active & $\begin{array}{l}\text { Three times measurement of BP using an } \\
\text { automatic oscillometric device }\end{array}$ \\
\hline $\begin{array}{l}\text { Kollias, 2009; } \\
\text { Greece [51] }\end{array}$ & Local & $\begin{array}{l}\text { Cross- } \\
\text { section }\end{array}$ & 1008 & 480 & $\begin{array}{l}12 \\
\text { to } \\
17\end{array}$ & $\begin{array}{l}\text { SBP } \\
\text { and } \\
\text { DBP }\end{array}$ & $\begin{array}{l}\text { At least } 1 \\
\text { cigarette per day }\end{array}$ & Active & $\begin{array}{l}\text { Three times measurement of BP using an } \\
\text { automatic oscillometric device }\end{array}$ \\
\hline $\begin{array}{l}\text { Lawlor, 2004; } \\
\text { Australia [52] }\end{array}$ & Local & Cohort & 3864 & NR & 5 & SBP & $\begin{array}{l}\text { Smoking at } 18 \\
\text { weeks gestation }\end{array}$ & Pregnancy & $\begin{array}{l}\text { Two times measurement of BP using an } \\
\text { digital sphygmomanometer device }\end{array}$ \\
\hline $\begin{array}{l}\text { Oken, 2005; USA } \\
\text { [53] }\end{array}$ & Local & Cohort & 746 & 373 & 3 & SBP & Current smoking & Pregnancy & $\begin{array}{l}\text { Up to } 5 \text { times measurement of BP using an } \\
\text { automatic oscillometric device }\end{array}$ \\
\hline $\begin{array}{l}\text { Rostand, 2005; } \\
\text { USA [54] }\end{array}$ & Local & $\begin{array}{l}\text { Cross- } \\
\text { section }\end{array}$ & 262 & 149 & 5 & SBP & NR & Pregnancy & $\begin{array}{l}\text { Single measurement of BP using a mercury } \\
\text { sphygmomanometer device }\end{array}$ \\
\hline $\begin{array}{l}\text { Simonetti, 2011; } \\
\text { Germany [42] }\end{array}$ & National & $\begin{array}{l}\text { Cross- } \\
\text { section }\end{array}$ & 4236 & 2181 & $\begin{array}{l}4 \\
\text { to } \\
7.5\end{array}$ & $\begin{array}{l}\text { SBP } \\
\text { and } \\
\text { DBP }\end{array}$ & Current smoking & $\begin{array}{l}\text { Pregnancy } \\
\text { and } \\
\text { domestic }\end{array}$ & $\begin{array}{l}\text { Three times measurement of BP using an } \\
\text { auscultatory aneroid sphygmomanometry } \\
\text { device }\end{array}$ \\
\hline $\begin{array}{l}\text { van den Berg, } \\
\text { 2013; Netherland [41] }\end{array}$ & Local & Cohort & 3024 & 1521 & $\begin{array}{l}5 \\
\text { to } \\
6\end{array}$ & $\begin{array}{l}\text { SBP } \\
\text { and } \\
\text { DBP }\end{array}$ & NR & $\begin{array}{l}\text { Pregnancy } \\
\text { and } \\
\text { domestic }\end{array}$ & $\begin{array}{l}\text { Two or three times measurement of } \mathrm{BP} \\
\text { using an automatic sphygmomanometer } \\
\text { device }\end{array}$ \\
\hline $\begin{array}{l}\text { Wen, 2011; USA } \\
\text { [55] }\end{array}$ & National & Cohort & 30,441 & 15,031 & 7 & SBP & $\begin{array}{l}\text { At least } 1 \\
\text { cigarette per day }\end{array}$ & Pregnancy & $\begin{array}{l}\text { Two times measurement of BP using a } \\
\text { digital oscillometric device }\end{array}$ \\
\hline $\begin{array}{l}\text { Yang, 2013; } \\
\text { Canada [56] }\end{array}$ & National & Cohort & 13,889 & 7173 & 6.5 & $\begin{array}{l}\text { SBP } \\
\text { and } \\
\text { DBP }\end{array}$ & $\begin{array}{l}\text { At least } 1 \\
\text { cigarette per day }\end{array}$ & $\begin{array}{l}\text { Pregnancy } \\
\text { and } \\
\text { domestic }\end{array}$ & $\begin{array}{l}\text { Single measurement of BP using a manual } \\
\text { sphygmomanometer device }\end{array}$ \\
\hline
\end{tabular}

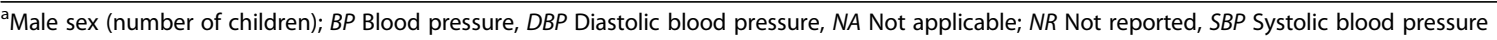

significantly affect absolute level of systolic blood pressure (pooled Beta $=0.01 ; 95 \%$ CI: -0.19 to 0.22 ). Publication bias was not observed in this section (coefficient $=5.21 ; p=0.38$ ).

Effect of passive exposure to cigarette smoke on absolute level of systolic blood pressure

Thirteen studies assessed the effect of passive exposure to cigarette smoke on absolute level of systolic blood pressure. After pooling the amounts of adjusted regression coefficients, it was shown that passive exposure to cigarette smoke leads to a significant increase in absolute level of systolic blood pressure (pooled coefficient $=0.26$; 95\% CI: 0.12 to 0.39 ) (Fig. 4). Heterogeneity was observed in this section (I2 = $50.4 \% ; p=0.004)$, but publication bias was not seen (coefficient $=3.98 ; p=0.06$ ). 


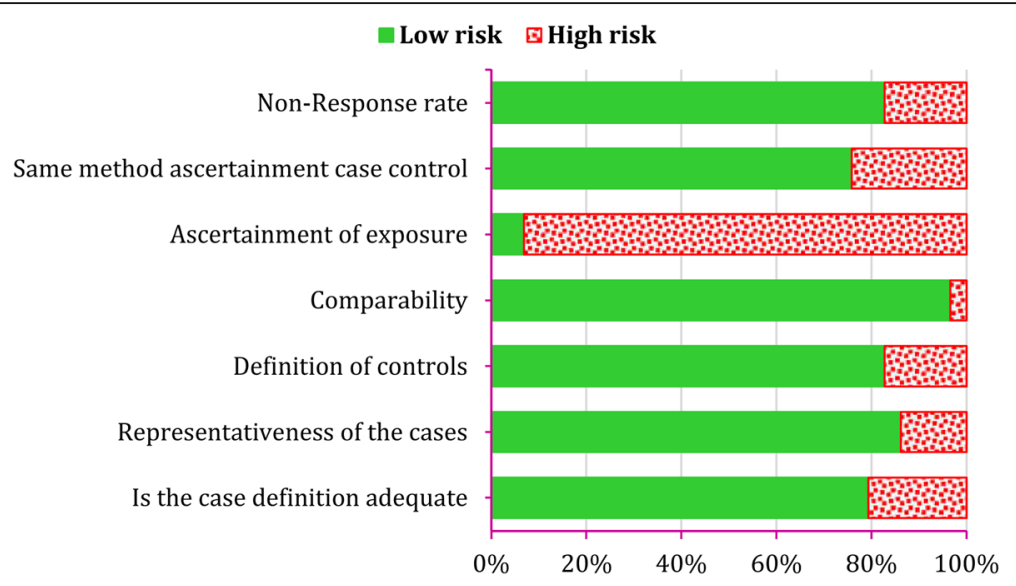

Fig. 2 Quality assessment of included studies according to Newcastle-Ottawa Scale assessment tools

Subgroup analysis showed that type of study, different age groups among children, different definitions of smoking, period of exposure and sample size were the most important causes of heterogeneity among studies. Pooled analysis of cohort studies showed that passive exposure to cigarette smoke increases absolute level of systolic blood pressure $(p<0.001)$; however, this association was not seen in cross-sectional studies $(p=0.44)$. Moreover, passive exposure in patients between the ages of 0 and 7 years old $(p<0.001)$ and 12 and 18 years old $(p=$ $0.001)$ was associated with higher levels of systolic blood pressure. In addition, passive exposure to cigarette smoke of individuals who are current daily smokers $(p=$ $0.003)$ or smoke at least one cigarette per week ( $p=$ 0.003 ) leads to an increase in absolute level of systolic blood pressure in children. Additionally, exposure to cigarette smoke during fetal period $(p<0.001)$ is also associated with an increase in absolute level of systolic blood pressure in childhood and adolescence (Table 4).

\section{Effect of active smoking on absolute level of diastolic blood pressure}

4 studies were entered in this section. Active smoking did not have a significant effect on absolute level of diastolic blood pressure (pooled coefficient $=0.01 ; 95 \% \mathrm{CI}$ : -0.18 to 0.20 ). Heterogeneity was observed in this section ( $\mathrm{I} 2=51.7 \% ; p=0.08)$, but publication bias was not seen (coefficient $=1.02 ; p=0.39$ ). The source of heterogeneity could not be found due to scarcity of studies (Fig. 4).

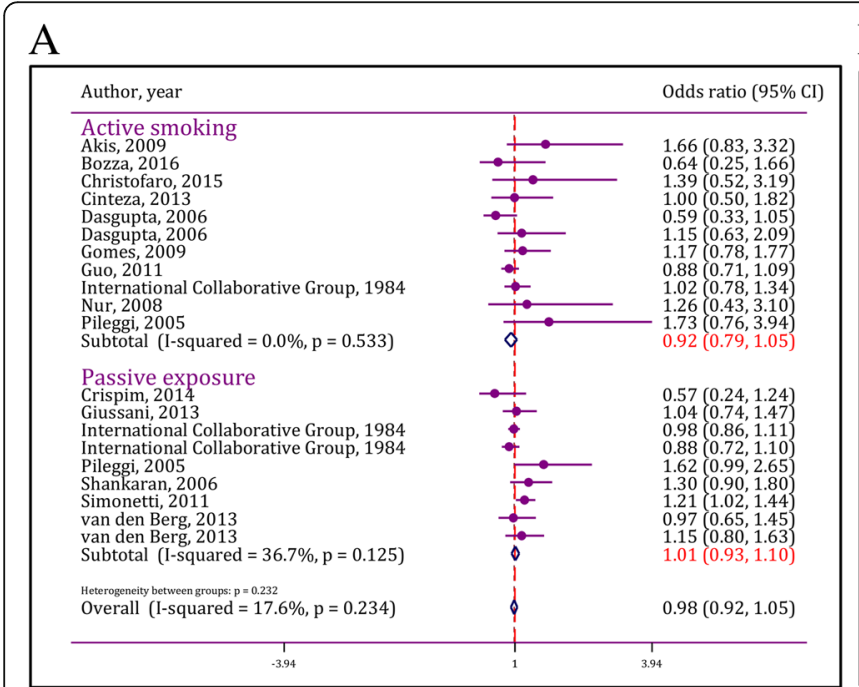

\section{B}

\begin{tabular}{|c|c|}
\hline Author, year & Odds ratio $(95 \% \mathrm{CI})$ \\
\hline \multicolumn{2}{|l|}{ Domestic exposure } \\
\hline Crispim, 2014 & $0.57(0.24,1.24)$ \\
\hline Giussani, 2013 & $1.04(0.74,1.47)$ \\
\hline Simonetti, 2011 & $1.21(1.02,1.44)$ \\
\hline van den Berg, 2013 & $1.15(0.80,1.63)$ \\
\hline Subtotal $(1-$ squared $=45.7 \%, p=0.137)$ & $1.05(0.81,1.29)$ \\
\hline \multicolumn{2}{|l|}{ Pregnancy } \\
\hline International Collaborative Group, 1984 & $0.98(0.86,1.11)$ \\
\hline International Collaborative Group, 1984 & $0.88(0.72,1.10)$ \\
\hline Pileggi, 2005 & $1.62(0.99,2.65)$ \\
\hline Shankaran, 2006 & $1.30(0.90,1.80)$ \\
\hline van den Berg, 2013 & $0.97(0.65,1.45)$ \\
\hline Subtotal (I-squared $=24.4 \%, p=0.259)$ & $0.99(0.85,1.13)$ \\
\hline Overall (I-squared $=36.7 \%, \mathrm{p}=0.125$ ) & $1.03(0.91,1.15)$ \\
\hline \multicolumn{2}{|l|}{ 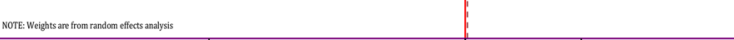 } \\
\hline-265 & 65 \\
\hline
\end{tabular}

Fig. 3 Forest plot of active and passive exposure to cigarette smoke in incidence of hypertension in children and adolescents A) Pooled odds ratio B) subgroup analysis of effect of passive exposure during pregnancy and domestic exposure on incidence of hypertension. Cl: Confidence interval 


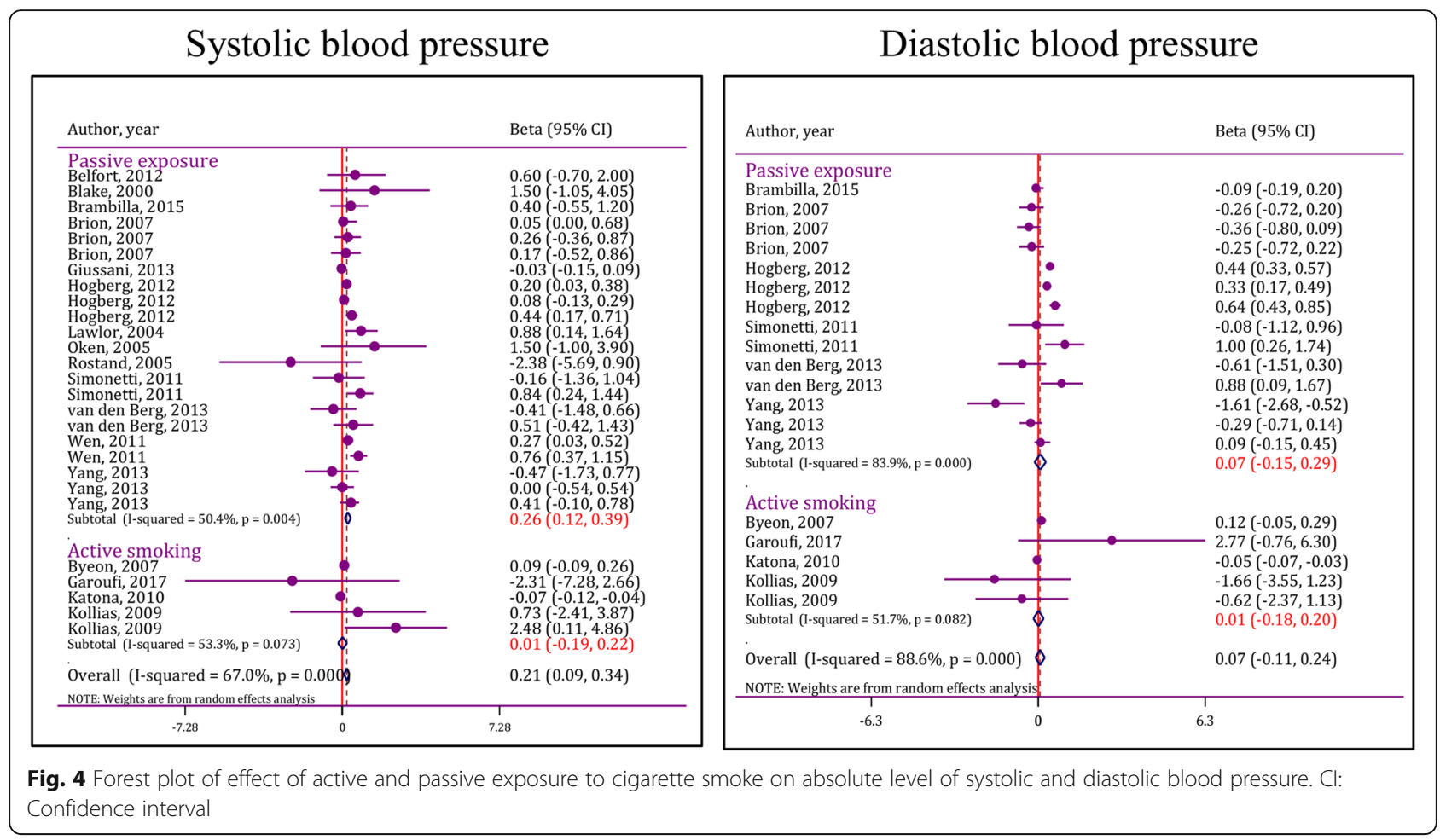

\section{Effect of passive exposure to cigarette smoke on absolute level of diastolic blood pressure}

6 studies assessed the effect of passive exposure to cigarette smoke on absolute level of diastolic blood pressure. Similar to active smoking, passive exposure to cigarette smoke did not have a significant effect on absolute level of diastolic blood pressure (pooled coefficient $=0.07 ; 95 \% \mathrm{CI}:-0.15$ to 0.29$)$. Heterogeneity was observed in this section (I2 $=83.9 \% ; p<0.001$ ), but publication bias was not seen (coefficient $=4.90$; $p=0.44)$. Subgroup analysis could not be done in this section due to scarcity of studies.

\section{Discussion}

For the first time, the present meta-analysis assessed the effect of active smoking or passive exposure to cigarette smoke on risk of developing hypertension in children and adolescents. Although analyses showed that active smoking or passive exposure to cigarette smoke were not associated with developing hypertension in children and adolescents, passive exposure to cigarette smoke was associated with higher levels of systolic blood pressure. In the present study, it was shown that passive exposure to cigarette smoke during fetal period increases the level of systolic blood pressure in childhood and adolescence.

The present meta-analysis showed that active smoking was not associated with developing hypertension or absolute level of blood pressure. The cause of this finding could be found in cumulative effect of cigarette smoking. While assessing cigarette consumption, duration of smoking is an influential factor which should be considered. Hence, the term "pack-year" is used in cigarette studies [57-61]. The mentioned term indicates number of cigarettes used and smoking duration. Adverse effects of cigarette smoking in children and adolescents may not be evident as duration of active smoking is short in this population. There was no study emphasizing on duration of active smoking among entered studies of the present meta-analysis. Therefore, subgroup analysis could not be done based on duration of consumption or exposure.

A longitudinal survey showed that there is no associations between smoking and the risk of hypertension in individuals younger than 35 years old; but smoking was significantly associated with hypertension in older ages [62]. Therefore, it seems that the duration of exposure to cigarette smoke is a potential covariate for assessment of smoking and hypertension. However, most of eligible studies in the current meta-analysis were cross-sectional with short follow-up periods. Therefore, the lack of a significant relationship between smoking and hypertension may be due to limitations of the included studies.

Passive smoking, mainly starting in the fetal period, has a longer duration in children and adolescents than active smoking, which tends to start later on, during adolescence. This issue may be an explanation for the absence of association between active smoking and 
Table 4 Subgroup analysis of smoking effects on pediatric systolic blood pressure

\begin{tabular}{|c|c|c|c|c|c|}
\hline Category & Model & Publication bias & Heterogeneity $^{a}$ & Beta $(95 \% \mathrm{Cl})$ & $P_{\text {for effect size }}$ \\
\hline \multicolumn{6}{|l|}{ Age group (year) } \\
\hline $0-7$ & FEM & $p=0.55$ & $33.0 \%(p=0.11)$ & 0.39 (0.24 to 0.55$)$ & $<0.001$ \\
\hline $7-13$ & FEM & $p=0.04$ & $0.0 \%(p=0.86)$ & $0.14(-0.12$ to 0.40$)$ & 0.31 \\
\hline $12-18$ & FEM & $p=0.68$ & $53.2 \%(p=0.12)$ & 0.21 (0.09 to 0.33 ) & 0.001 \\
\hline \multicolumn{6}{|l|}{ Type of study } \\
\hline Cohort & FEM & $p=0.04$ & $27.2 \%(p=0.14)$ & 0.25 (0.16 to 0.34$)$ & $<0.001$ \\
\hline Cross-sectional & REM & $p=0.68$ & $62.5 \%(p=0.03)$ & $0.21(-0.32$ to 0.74$)$ & 0.44 \\
\hline \multicolumn{6}{|l|}{ Smoking definition } \\
\hline Not reported & FEM & $p=0.88$ & $28.8 \%(p=0.24)$ & $0.16(-0.38$ to 0.70$)$ & 0.57 \\
\hline At least 1 cigarette per month & FEM & $p=0.47$ & $29.0 \%(p=0.21)$ & 0.02 (-0.09 to 0.13$)$ & 0.71 \\
\hline Current daily smoking & FEM & $p=0.83$ & $45.7 \%(p=0.14)$ & 0.25 (0.08 to 0.41$)$ & 0.003 \\
\hline At least 1 cigarette per month & REM & $p=0.82$ & $55.3 \%(p=0.004)$ & $0.30(0.10$ to 0.50$)$ & 0.003 \\
\hline \multicolumn{6}{|l|}{ Period of exposure } \\
\hline Pregnancy & REM & $p=0.02$ & $35.4 \%(p=0.08)$ & $0.26(0.12$ to 0.41$)$ & $<0.001$ \\
\hline Domestic (postnatal) & REM & $p=0.07$ & $59.8 \%(p=0.03)$ & $0.28(-0.04$ to 0.59$)$ & 0.08 \\
\hline \multicolumn{6}{|l|}{ Parental smoking habit } \\
\hline Mother & REM & $p=0.04$ & $43.2 \%(p=0.04)$ & 0.25 (0.09 to 0.41$)$ & 0.002 \\
\hline Father & NA & NA & NA & NA & NA \\
\hline Both & REM & $p=0.05$ & $56.8 \%(p=0.03)$ & 0.34 (0.01 to 0.67 ) & 0.04 \\
\hline \multicolumn{6}{|l|}{ Sample size } \\
\hline$<1000$ subjects & FEM & $p=0.91$ & $27.5 \%(p=0.25)$ & 0.61 (-0.41 to 1.63$)$ & 0.24 \\
\hline$\geq 1000$ subjects & REM & $p=0.07$ & $54.6 \%(p=0.003)$ & 0.25 (0.11 to 0.39$)$ & $<0.001$ \\
\hline \multicolumn{6}{|l|}{ BP measurement device } \\
\hline Mercury/aneroid & REM & 0.576 & $56.5 \%(p=0.011)$ & 0.11 (0.03 to 0.20$)$ & 0.007 \\
\hline Automatic/semiautomatic & FEM & 0.257 & $26.7 \%(p=0.190)$ & 0.33 (0.17 to 0.48$)$ & $<0.001$ \\
\hline
\end{tabular}

${ }^{a}$ Heterogeneity was reported as I-squared and corresponding $\mathrm{p}$ value. Cl Confidence interval, FEM Fixed effect model, NA Not applicable due to lack of included studies, REM Random effect mode

blood pressure level. Therefore, it is suggested to assess a life-course association of smoking and hypertension in future studies.

Subgroup analysis was done to assess the association between passive exposure to cigarette smoke and absolute level of systolic blood pressure due to presence of significant heterogeneity among related studies. Different definitions of smoking among studies were the most important source of heterogeneity. There was a significant association between passive exposure to cigarette smoke and absolute level of systolic blood pressure in studies which smoking was defined as number of cigarettes smoked per day or week. However, a significant association was not seen in studies which used non-standard definitions such as "smoker or non-smoker". Overall, definition of smoking was diverse among studies. Therefore, it is possible that some cases are wrongly put in smoker group and hence explaining the non-significant association seen between cigarette smoking and blood pressure.
Effect of cigarette smoking in parents during pregnancy on absolute level of systolic blood pressure in childhood and adolescence was one of the most important findings of the present study. Absolute levels of blood pressure were higher in children who their parents especially their mothers had a history of cigarette smoking. The cause of mentioned finding might be due to the effect of harmful substances present in cigarette smoke on fetal growth [44]. This finding shows that although active or passive exposure to cigarette smoke does not lead to development of hypertension in children and adolescence, it results in higher levels of absolute blood pressure in this age group. The importance of this finding is that elevated level of absolute blood pressure in childhood is a known risk factor for hypertension during adulthood. Hence, these children might get hypertension during adulthood [63-66].

Although blood pressure measurement methods were slightly different among studies, most of them used the standard protocol for BP measurement. Apart from two 
articles, other studies attempted to measure blood pressure at least 2 times and included the mean of these two values in their analyses. The only major diversity among eligible studies was the device used to measure blood pressure. 11 studies used mercury or aneroid sphygmomanometer devices while 18 studies used automatic oscillometric devices. Subgroup analysis showed that the type of blood pressure measurement device does not affect the relationship between smoking and systolic blood pressure value. Therefore, it seems that the method of measuring blood pressure does not affect the findings of this study.

\section{Limitations}

High level of heterogeneity among studies was one of limitations of the present study. Different definitions of smoking were the most important source of heterogeneity and led to use of random effect analysis in order to present a more conservative effect size. Definition of smoking was not standard in many studies as many studies which were highly focused on cigarette smoking defined smoking as consumption of at least 100 cigarettes [67-69]. However, the mentioned definition was not used in any of entered studies. In many studies cigarette smoking was defined as consumption of at least 1 cigarette per day, but this definition may be biased due to lack of information about duration of smoking. Follow up period was diverse among studies as researchers of the present study could not categorize studies according to their follow-up period for further assessments. Additionally, adjusting for confounders in order to assess reported associations had a high diversity in different studies. Some of them had entered socio-economic and socio-demographic factors in their models while they were not entered in other studies. Therefore, difference in adjustments might be another factor influencing results.

\section{Conclusion}

The present study showed that both active and passive cigarette smoking were not associated with developing hypertension in children and adolescents. However, exposure to passive cigarette smoke was associated with higher level of systolic blood pressure in children and adolescents.

\section{Abbreviations}

BP: Blood pressure; Cl: Confidence interval; HTN: Hypertension; NA: Not applicable; NR: Not reported

\section{Acknowledgments}

Not applicable.

\section{Funding}

Not applicable.

\section{Availability of data and materials}

The datasets used and/or analysed during the current study are available from the corresponding author on reasonable request.

\section{Authors' contributions}

$M A, M Y, M H$ and $H J$ designed the study. MY, MA and $A O$ participated in acquisition of data. $\mathrm{MH}$ and $\mathrm{GH}$ analyzed the data. MK and $\mathrm{HS}$ participate in management of data. MY and $\mathrm{AO}$ wrote the first draft and other revising manuscript critically. All authors approved final version of the manuscript to be published and are accountable for all aspects of the work.

\section{Ethics approval and consent to participate}

The study designs were approved by Tehran University of Medical Sciences Ethics Committee. In this study an informed consent was not applicable.

\section{Consent for publication}

Not applicable.

\section{Competing interests}

The authors declare that they have no competing interests.

\section{Publisher's Note}

Springer Nature remains neutral with regard to jurisdictional claims in published maps and institutional affiliations.

\section{Author details \\ ${ }^{1}$ Tobacco Prevention and Control Research Center, National Research Institute of Tuberculosis and Lung Diseases (NRITLD), Shahid Beheshti University of Medical Sciences, Tehran, Iran. ${ }^{2}$ Physiology Research Center, Faculty of Medicine, Iran University of Medical Sciences, Tehran, Iran. ${ }^{3}$ Department of Medicine, Tehran University of Medical Sciences, Tehran, Iran. ${ }^{4}$ Mycobacteriology Research Center, Biostatistics Unit, NRITLD, Shahid Beheshti University of Medical Sciences, Tehran, Iran. ${ }^{5}$ Department of Epidemiology and Biostatistics, School of Public Health, Tehran University of Medical Sciences, Poursina Ave, Tehran, Iran.}

Received: 26 December 2018 Accepted: 10 April 2019

Published online: 21 May 2019

\section{References}

1. Bremner AD. Antihypertensive medication and quality of life-silent treatment of a silent killer? Cardiovasc Drugs Ther. 2002;16(4):353-64.

2. Adrogué HE, Sinaiko AR. Prevalence of hypertension in junior high schoolaged children: effect of new recommendations in the 1996 updated task force report. Am J Hypertens. 2001;14(5):412-4.

3. Arar MY, Hogg RJ, Arant BS Jr, Seikaly MG. Etiology of sustained hypertension in children in the southwestern United States. Pediatr Nephrol. 1994;8(2):186-9.

4. Stanley JC, Fry WJ. Pediatric renal artery occlusive disease and renovascular hypertension: etiology, diagnosis, and operative treatment. Arch Surg. 1981; 116(5):669-76.

5. Ferguson MA, Flynn JT. Treatment of pediatric hypertension: lessons learned from recent clinical trials. Current Cardiovascular Risk Reports. 2014;8(9):1-7.

6. Heydari G, Yousefifard M, Hosseini M, Ramezankhani A, Masjedi MR. Cigarette smoking, knowledge, attitude and prediction of smoking between male students, teachers and clergymen in Tehran, Iran, 2009. Int J Prev Med. 2013;4(5):557-64.

7. Barker DJ. Fetal origins of coronary heart disease. BMJ: British Medical Journal. 1995:311(6998):171.

8. Heydari G, Heidari F, Yousefifard M, Hosseini M. Smoking and diet in healthy adults: a cross-sectional study in Tehran, Iran, 2010. Iran J Public Health. 2014;43(4):485-91.

9. Beyerlein A, Rückinger $S$, Toschke AM, Rosario AS, von Kries R. Is low birth weight in the causal pathway of the association between maternal smoking in pregnancy and higher BMI in the offspring? Eur J Epidemiol. 2011;26(5): 413-20

10. Celermajer DS, Adams MR, Clarkson P, Robinson J, McCredie R, Donald A, et al. Passive smoking and impaired endothelium-dependent arterial dilatation in healthy young adults. N Engl J Med. 1996;334(3):150-5.

11. Kelishadi R, Noori A, Qorbani M, Rahimzadeh S, Djalalinia S, Shafiee G, et al. Are active and passive smoking associated with cardiometabolic risk factors 
in adolescents? The CASPIAN-III study. Paediatrics and international child health. 2016:1-8.

12. Lee I-M, Shiroma EJ, Lobelo F, Puska P, Blair SN, Katzmarzyk PT, et al. Effect of physical inactivity on major non-communicable diseases worldwide: an analysis of burden of disease and life expectancy. Lancet. 2012;380(9838): 219-29.

13. Hansson P-O, Eriksson H, Welin L, Svärdsudd K, Wilhelmsen L. Smoking and abdominal obesity: risk factors for venous thromboembolism among middle-aged men: the study of men born in 1913. Arch Intern Med. 1999; 159(16):1886-90.

14. Stroup DF, Berlin JA, Morton SC, Olkin I, Williamson GD, Rennie D, et al. Meta-analysis of observational studies in epidemiology. JAMA. 2000;283(15): 2008-12.

15. Ghelichkhani P, Yousefifard M, Nazemi L, Safari S, Hosseini M, Baikpour M, et al. The value of serum $\beta$-subunit of human chorionic gonadotropin level in prediction of treatment response to methotrexate in management of ectopic pregnancy; a systematic review and meta-analysis. International Journal of Pediatrics. 2016;4(9):3503-18.

16. Hassannejad Z, Yousefifard M, Azizi Y, Zadegan SA, Sajadi K, SharifAlhoseini $\mathrm{M}$, et al. Axonal degeneration and demyelination following traumatic spinal cord injury: a systematic review and meta-analysis. Chem Neuroanat. 2019;97:9-22.

17. Yousefifard M, Sarveazad A, Babahajian A, Baikpour M, Shokraneh F, Vaccaro $A R$, et al. Potential diagnostic and prognostic value of serum and cerebrospinal fluid biomarkers in traumatic spinal cord injury: a systematic review. J Neurochem. 2018.

18. Nakhjavan-Shahraki B, Yousefifard M, Rahimi-Movaghar V, Baikpour M, Nasirinezhad F, Safari S, et al. Transplantation of olfactory ensheathing cells on functional recovery and neuropathic pain after spinal cord injury; systematic review and meta-analysis. Sci Rep. 2018:8(1):325.

19. Nakhjavan-Shahraki B, Yousefifard M, Ataei N, Baikpour M, Ataei F, Bazargani $B$, et al. Accuracy of cystatin $C$ in prediction of acute kidney injury in children; serum or urine levels: which one works better? A systematic review and meta-analysis. BMC Nephrol. 2017;18(1):120.

20. Safari S, Najafi I, Hosseini M, Baratloo A, Yousefifard M, Mohammadi H. 20day trend of serum potassium changes in bam earthquake victims with crush syndrome; a cross-sectional study. Emergency. 2017;5(1).

21. Hashemi B, Safari S, Hosseini M, Yousefifard M, Erfani E, Baratloo A, et al. A systematic review of Iranian experiences in seismo-nephrology. Archives of trauma research. 2016;5(2)

22. Nakhjavan-Shahraki B, Yousefifard M, Oraii A, Sarveazad A, Hosseini M. Metaanalysis of neuron specific enolase in predicting pediatric brain injury outcomes. EXCLI J. 2017:16:995-1008.

23. Izadi A, Yousefifard M, Nakhjavan-Shahraki B, Baikpour M, Mirzay Razaz J, Hosseini M. Diagnostic value of urinary neutrophil gelatinaseassociated Lipocalin (NGAL) in detection of pediatric acute kidney injury; a systematic review and meta-analysis. International Journal of Pediatrics. 2016:4(11):3875-95

24. Izadi A, Yousefifard M, Nakhjavan-Shahraki B, Baikpour M, Mirzay Razaz J, Ataei $\mathrm{N}$, et al. Value of plasma/serum neutrophil gelatinase-associated lipocalin in detection of pediatric acute kidney injury; a systematic review and meta-analysis. International Journal of Pediatrics. 2016;4(11): 3815-36.

25. Moher D, Liberati A, Tetzlaff J, Altman DG. Preferred reporting items for systematic reviews and meta-analyses: the PRISMA statement. Ann Intern Med. 2009;151(4):264-9.

26. Sistrom $\mathrm{CL}$, Mergo PJ. A simple method for obtaining original data from published graphs and plots. Am J Roentgenol. 2000;174(5):1241-4.

27. Stang A. Critical evaluation of the Newcastle-Ottawa scale for the assessment of the quality of nonrandomized studies in meta-analyses. Eur J Epidemiol. 2010;25(9):603-5.

28. Akis N, Pala K, Meric-Utku A, Seyithan B, Sarandol E, Aytekin H. Hypertension in children (12-14 years)--a case-control study in Bursa. Turkey Turk J Pediatr. 2009:51(5):437-43.

29. Bozza R, Campos W, Barbosa Filho VC, Stabelini Neto A, Silva MP, Maziero RS. High blood pressure in adolescents of Curitiba: prevalence and associated factors. Arq Bras Cardiol. 2016;106(5):411-8.

30. Christofaro DG, De Andrade SM, Cardoso JR, Mesas AE, Codogno JS, Fernandes RA. High blood pressure and sedentary behavior in adolescents are associated even after controlling for confounding factors. Blood Press. 2015;24(5):317-23.
31. Cinteza $E$, Balgradean M. Hypertension in romanian children and adolescents: a cross-sectional survey. Maedica (Buchar). 2013;8(1):5-10.

32. Crispim PA, Peixoto Mdo R, Jardim PC. Risk factors associated with high blood pressure in two-to five-year-old children. Arq Bras Cardiol. 2014;102(1):39-46.

33. Dasgupta K, O'Loughlin J, Chen S, Karp I, Paradis G, Tremblay J, et al. Emergence of sex differences in prevalence of high systolic blood pressure: analysis of a longitudinal adolescent cohort. Circulation. 2006;114(24):2663-70.

34. Giussani M, Antolini L, Brambilla P, Pagani M, Zuccotti G, Valsecchi MG, et al. Cardiovascular risk assessment in children: role of physical activity, family history and parental smoking on BMI and blood pressure. J Hypertens. 2013; 31(5):983-92.

35. Gomes Bda M, Alves JG. Prevalence of high blood pressure and associated factors in students from public schools in greater metropolitan Recife, Pernambuco state, Brazil, 2006. Cad Saude Publica. 2009;25(2):375-81.

36. Guo X, Zhang X, Li Y, Zhou X, Yang H, Ma H, et al. Differences in healthy lifestyles between prehypertensive and normotensive children and adolescents in northern China. Pediatr Cardiol. 2012;33(2):222-8.

37. International Collaborative Group. International collaborative study on juvenile hypertension. 2. First follow-up report. International Collaborative Group. Bull World Health Organ. 1984;62(1):121-32.

38. Nur N, Cetinkaya S, Yilmaz A, Ayvaz A, Bulut MO, Sumer H. Prevalence of hypertension among high school students in a middle Anatolian province of Turkey. J Health Popul Nutr. 2008;26(1):88-94.

39. Pileggi C, Carbone V, Nobile CG, Pavia M. Blood pressure and related cardiovascular disease risk factors in 6-18 year-old students in Italy. J Paediatr Child Health. 2005:41(7):347-52.

40. Shankaran S, Das A, Bauer CR, Bada H, Lester B, Wright L, et al. Fetal origin of childhood disease: intrauterine growth restriction in term infants and risk for hypertension at 6 years of age. Archives of pediatrics \& adolescent medicine. 2006:160(9):977-81.

41. van den Berg G, van Eijsden M, Galindo-Garre F, Vrijkotte TG, Gemke RJ. Explaining socioeconomic inequalities in childhood blood pressure and prehypertension: the ABCD study. Hypertension. 2013;61(1):35-41.

42. Simonetti GD, Schwertz R, Klett M, Hoffmann GF, Schaefer F, Wuhl E. Determinants of blood pressure in preschool children: the role of parental smoking. Circulation. 2011:123(3):292-8.

43. Belfort MB, Gillman MW, McCormick MC. Prenatal and perinatal predictors of blood pressure at school age in former preterm, low birth weight infants. J Perinatol. 2012;32(4):265-9.

44. Blake KV, Gurrin LC, Evans SF, Beilin LJ, Landau LI, Stanley FJ, et al. Maternal cigarette smoking during pregnancy, low birth weight and subsequent blood pressure in early childhood. Early Hum Dev. 2000;57(2):137-47.

45. Brambilla P, Bedogni G, Pietrobelli A, Cianfarani S, Agostoni C. Predictors of blood pressure at 7-13 years: the "new millennium baby" study. Nutr Metab Cardiovasc Dis. 2016;26(8):706-12.

46. Brion MJ, Leary SD, Smith GD, Ness AR. Similar associations of parental prenatal smoking suggest child blood pressure is not influenced by intrauterine effects. Hypertension. 2007;49(6):1422-8.

47. Byeon YS, Lee HS. Relation of the blood pressure, lipids and body mass index by smoking status among adolescents. Taehan Kanho Hakhoe Chi. 2007:37(6):1020-6.

48. Garoufi A, Grammatikos EE, Kollias A, Grammatikos E, Stergiou GS, Soldatou A. Associations between obesity, adverse behavioral patterns and cardiovascular risk factors among adolescent inhabitants of a Greek island. J Pediatr Endocrinol Metab. 2017:30(4):445-54.

49. Hogberg L, Cnattingius S, Lundholm C, D'Onofrio BM, Langstrom N, Iliadou AN. Effects of maternal smoking during pregnancy on offspring blood pressure in late adolescence. J Hypertens. 2012;30(4):693-9.

50. Katona E, Zrinyi M, Komonyi E, Lengyel S, Paragh G, Zatik J, et al. Factors influencing adolescent blood pressure: the Debrecen hypertension study. Kidney Blood Press Res. 2011;34(3):188-95.

51. Kollias A, Antonodimitrakis P, Grammatikos E, Chatziantonakis N, Grammatikos EE, Stergiou GS. Trends in high blood pressure prevalence in Greek adolescents. J Hum Hypertens. 2009;23(6):385-90.

52. Lawlor DA, Najman JM, Sterne J, Williams GM, Ebrahim S, Davey SG. Associations of parental, birth, and early life characteristics with systolic blood pressure at 5 years of age: findings from the Mater-University study of pregnancy and its outcomes. Circulation. 2004;110(16):2417-23.

53. Oken E, Huh SY, Taveras EM, Rich-Edwards JW, Gillman MW. Associations of maternal prenatal smoking with child adiposity and blood pressure. Obes Res. 2005;13(11):2021-8. 
54. Rostand SG, Cliver SP, Goldenberg RL. Racial disparities in the association of foetal growth retardation to childhood blood pressure. Nephrol Dial Transplant. 2005;20(8):1592-7.

55. Wen X, Triche EW, Hogan JW, Shenassa ED, Buka SL. Prenatal factors for childhood blood pressure mediated by intrauterine and/or childhood growth? Pediatrics. 2011;127(3):e713-21.

56. Yang S, Decker A, Kramer MS. Exposure to parental smoking and child growth and development: a cohort study. BMC Pediatr. 2013;13:104.

57. Aryanpur M, Masjedi MR, Mortaz E, Hosseini M, Jamaati H, Tabarsi P, et al. Intention to quit smoking and associated factors in smokers newly diagnosed with pulmonary tuberculosis. Tanaffos. 2016;15(1):17-24.

58. Aryanpur M, Hosseini M, Masjedi MR, Mortaz E, Tabarsi P, Soori H, et al. A randomized controlled trial of smoking cessation methods in patients newly-diagnosed with pulmonary tuberculosis. BMC Infect Dis. 2016;16:369.

59. Masjedi MR, Hosseini M, Aryanpur M, Mortaz E, Tabarsi P, Soori H, et al. The effects of smoking on treatment outcome in patients newly diagnosed with pulmonary tuberculosis. Int J Tuberc Lung Dis. 2017;21(3):351-6.

60. Aryanpur M, Masjedi MR, Hosseini M, Mortaz E, Tabarsi P, Soori H, et al. Cigarette smoking in patients newly diagnosed with pulmonary tuberculosis in Iran. Int J Tuberc Lung Dis. 2016;20(5):679-84.

61. Mohd Shariff N, Shah SA, Kamaludin F. Previous treatment, sputum-smear nonconversion, and suburban living: the risk factors of multidrug-resistant tuberculosis among Malaysians. Int J Mycobacteriol. 2016;5(1):51-8.

62. Gao K, Shi X, Wang W. The life-course impact of smoking on hypertension, myocardial infarction and respiratory diseases. Scientific reports. 2017;7(1):4330-

63. Sun SS, Grave GD, Siervogel RM, Pickoff AA, Arslanian SS, Daniels SR. Systolic blood pressure in childhood predicts hypertension and metabolic syndrome later in life. Pediatrics. 2007;119(2):237-46.

64. Klumbiene J, Sileikiene L, Milasauskiene Z, Zaborskis A, Shatchkute A. The relationship of childhood to adult blood pressure: longitudinal study of juvenile hypertension in Lithuania. J Hypertens. 2000;18(5):531-8.

65. Lurbe E. Childhood blood pressure: a window to adult hypertension. Hypertens. 2003;21(11):2001-3.

66. Chen W, Srinivasan SR, Ruan L, Mei H, Berenson GS. Adult hypertension is associated with blood pressure variability in childhood in blacks and whites: the Bogalusa heart study. Am J Hypertens. 2011;24(1):77-82.

67. Agaku IT, King BA, Dube SR. Control CfD, prevention. Current cigarette smoking among adults_-United States, 2005-2012. MMWR Morb Mortal Wkly Rep. 2014;63(2):29-34

68. Bondy SJ, Victor JC, Diemert LM. Origin and use of the 100 cigarette criterion in tobacco surveys. Tob Control. 2009;18(4):317-23.

69. Ahmad AM, Akhtar S, Hasan R, Khan JA, Hussain SF, Rizvi N. Risk factors for multidrug-resistant tuberculosis in urban Pakistan: a multicenter casecontrol study. Int J Mycobacteriol. 2012;1(3):137-42.

Ready to submit your research? Choose BMC and benefit from:

- fast, convenient online submission

- thorough peer review by experienced researchers in your field

- rapid publication on acceptance

- support for research data, including large and complex data types

- gold Open Access which fosters wider collaboration and increased citations

- maximum visibility for your research: over $100 \mathrm{M}$ website views per year

At $\mathrm{BMC}$, research is always in progress.

Learn more biomedcentral.com/submissions 